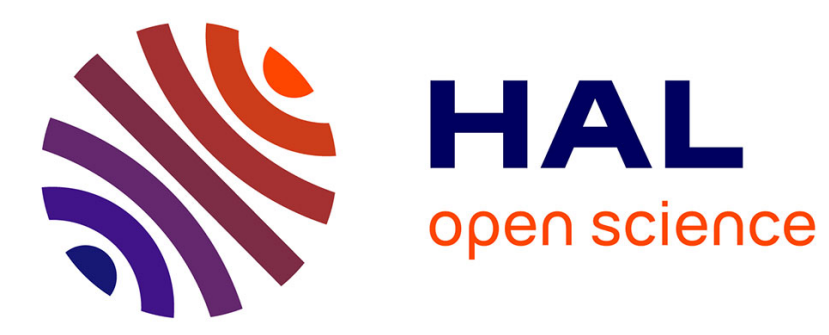

\title{
3D simulation of laminated shell structures using the Proper Generalized Decomposition
}

\author{
Etienne Pruliere
}

\section{To cite this version:}

Etienne Pruliere. 3D simulation of laminated shell structures using the Proper Generalized Decomposition. Composite Structures, 2014, 117, pp.373-381. 10.1016/j.compstruct.2014.06.039 . hal01062890

\section{HAL Id: hal-01062890 \\ https://hal.science/hal-01062890}

Submitted on 10 Sep 2014

HAL is a multi-disciplinary open access archive for the deposit and dissemination of scientific research documents, whether they are published or not. The documents may come from teaching and research institutions in France or abroad, or from public or private research centers.
L'archive ouverte pluridisciplinaire HAL, est destinée au dépôt et à la diffusion de documents scientifiques de niveau recherche, publiés ou non, émanant des établissements d'enseignement et de recherche français ou étrangers, des laboratoires publics ou privés. 


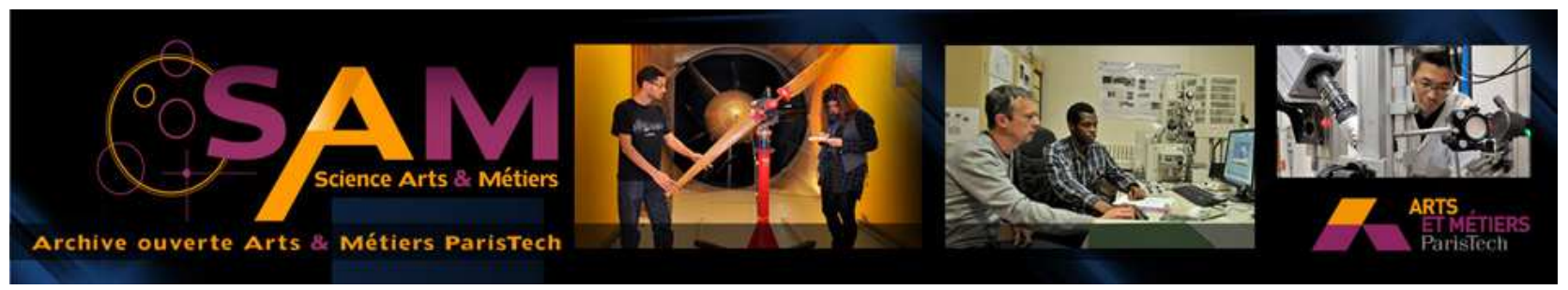

Science Arts \& Métiers (SAM)

is an open access repository that collects the work of Arts et Métiers ParisTech researchers and makes it freely available over the web where possible.

This is an author-deposited version published in: http://sam.ensam.eu Handle ID: .http://hdl.handle.net/10985/8489

\section{To cite this version :}

Etienne PRULIERE - 3D simulation of laminated shell structures using the Proper Generalized Decomposition - Composite Structures - Vol. 117, p.373-381 - 2014 


\title{
3D simulation of laminated shell structures using the Proper Generalized Decomposition
}

\author{
E. Prulière \\ Arts et Métiers ParisTech, I2M, UMR 5295, F-33400 Talence, France
}

Keywords:

Proper Generalized Decomposition

Shell model

Composite laminates

Model reduction

\begin{abstract}
A B S T R A C T
Numerical simulations of composite structures are generally performed using multi-layered shell elements in the context of the finite elements method. This strategy has numerous advantages like a low computation time and the capability to reproduce the comportment of composites in most of cases. The main restriction of this approach is that they require an approximation of the comportment in the thickness. This approximation is generally no more valid near the boundary and loading conditions and when non linear phenomena like delamination occurs in the thickness. This paper explores an alternative to shell computation using the framework of the Proper Generalized Decomposition that is based on a separated representation of the solution. The idea is to solve the full 3D solid problem separating the in-plane and the out-of-plane spaces. Practically, a classical shell mesh is used to describe the in-plane geometry and a simple 1D mesh is used to deal with the out-of-plane space. This allows to represents complex fields in the thickness without the complexity and the computation cost of a solid mesh which is particularly interesting when dealing with composite laminates.
\end{abstract}

\section{Introduction}

Most of shell structures simulations are based on the shell theory. A 3D shell structure is described from its mid-plane surface adding variables (angular coordinates in the classic shell theory) to treat the comportment in the thickness. Many models have been developed for multilayered structures: some use the Equivalent Single Layer approach where the variables concern the whole laminate and others use a Layerwise approach where the layers are described with different variables (see $[17,4]$ for a detailed review of these kinds of models). Shell models help reducing the computational cost required by full 3D finite element modeling but they require an approximation of the comportment in the thickness. The shell approximation is generally no more valid when increasing the thickness, near the boundary and loading conditions and when non linear phenomena like delamination occurs in the thickness. Another restriction is that the loads are applied on the mid-plane surface with no distinctions between a load applied on the top and on the bottom of the structures. However, this kind of models is largely widespread because it has proved its predictive ability in many cases and because of the difficulty to use full 3D strategies. The number of degrees of freedom needed to perform a full 3D finite elements simulation is damning. If one wants to keep a sufficient number of nodes in the thickness to allow a good precision,

E-mail address: e.pruliere@i2m.u-bordeaux1.fr he will be constrained to add an important number of nodes in the mid-plane surface in order to avoid bad quality elongated elements. This becomes quickly unmanageable.

An alternative to shell elements for reducing the computational time is to use model reduction strategies based on a separated representation of the solution. For example, a separated representation of a function $u$ defined on a 2D domain is:

$u(x, y) \approx \sum_{i=1}^{N} F_{i}(x) G_{i}(y)$

We can distinguish two approaches to build this separated representation.

The first one is to postulate a set of bases functions defined on a specified space (for example the space generated by $y$ ) and to solve the problem over the orthogonal complement (for example the space defined by $x$ ) considering this restricted number of bases functions. This strategy has been initially introduced in fluid mechanics [12] and has been widely used for space-time problems where the basis is set over the spacial domain. The number of basis functions is not related to the number of nodes like in the classic finite element method and is generally very restricted. The basis functions are determined "a posteriori" using for instance some results coming from a full computation or experimental data or just postulated from physic. The most common way to extract the basis functions from data is to perform a Proper Orthogonal Decomposition (POD) which gives the most significant modes. 
This strategy is close to shell finite elements modeling in the fact that the solution is postulated over one subspace (describing the thickness in the case of shell elements). It is possible to build a set of basis function defined in the thickness that represents rigid rotation and membrane displacement and to solve the problem in the orthogonal complement of this subspace (the mid-plane surface). This strategy leads to a solution similar to the one given by the classic shell theory with a similar computational cost.

The second approach is to build the separated representation "a priori" i.e. without making any approximation $[19,20]$. An appealing method is the A Priori Hyper Reduction method (APHR). It consists in controlling and enriching the basis only if needed. This techniques is particularly well adapted to treat space/time problems because the validity of the basis can be checked on some chosen time steps.

Another method lies in the use of the Proper Generalized Decomposition (PGD). The algorithm builds the reduced separated approximation and solves the problem at the same time. It may be applied for multi-dimensional problems and can treat a high variety of problems. This method has been introduced by Ladeveze with space-time problems in the context of the LATIN method under the name of "radial approximation" [10,11]. It has been generalized for multi-dimensional problems with some applications in models encountered in the kinetic theory description of complex fluids (Ammar et al. [1,2], Mokdad et al. [13]). It has also been applied for thermal problems $[7,16]$ and for parametric mechanical problems [6,14]. Recently, Vidal et al. [21] have used the PGD to simulate beam structures separating the axis of the beam from the section. The PGD has also been successfully applied to treat plate structures in [3] in the case of rectangular parallelepiped domains. Until now, the PGD approach has been mainly restricted in rectangular domains for 2D problems or hyper-rectangular domains for higher dimensional problems. This is due to the separated representation. In the case of non hyper-rectangular domains, a fully separated representation has been performed [9] but it involves some technical points and leads to a loss of overall performance. So the PGD method is a priori not well adapted to model complex shell structures.

The idea of this article is to use the PGD method to perform simulations of shell structures with complex shapes and curvatures without any a priori knowledge of the comportment in the thickness and using the PGD efficiency.

\section{A reduced strategy adapted to shell structures mechanical simulation}

\subsection{Mechanical model}

The model used is based on the classical momentum conservation equation:

$\operatorname{div} \boldsymbol{\sigma}+\mathbf{f}=\rho \boldsymbol{\Gamma}$

where $\boldsymbol{\sigma}$ denotes the stress tensor, div denotes the tensorial divergence, $\mathbf{f}$ is the volume force, $\rho$ is the density and $\boldsymbol{\Gamma}$ is the acceleration. $\boldsymbol{\sigma}$ is linked to the deformation by the constitutive relation:

$\boldsymbol{\sigma}=\mathbf{H} \boldsymbol{\varepsilon}$

where $\boldsymbol{\varepsilon}$ is the strain tensor and $\mathbf{H}$ is the fourth order rigidity tensor. This relation can be written using matrix notations:

$$
\left[\begin{array}{c}
\sigma_{x x} \\
\sigma_{y y} \\
\sigma_{z z} \\
\sigma_{y z} \\
\sigma_{x z} \\
\sigma_{x y}
\end{array}\right]=\mathbf{H}\left[\begin{array}{c}
\varepsilon_{x x} \\
\varepsilon_{y y} \\
\varepsilon_{z z} \\
2 \varepsilon_{y z} \\
2 \varepsilon_{x z} \\
2 \varepsilon_{x y}
\end{array}\right]
$$

$\boldsymbol{\varepsilon}$ is the symmetric gradient of the displacement $\mathbf{u}=(u, v, w)$.

The weak formulation of the equilibrium equation Eq. (2) without dynamic effect is:

$\int_{\Omega} \boldsymbol{\varepsilon}\left(\mathbf{u}^{\star}\right):(\mathbf{H} \boldsymbol{\varepsilon}(\mathbf{u}))=\int_{\Omega} \mathbf{u}^{\star} \cdot \mathbf{f}+\int_{\partial \Omega} \mathbf{u}^{\star} \cdot(\boldsymbol{\sigma} \cdot \mathbf{n})$

$\Omega$ is the domain taken by the structure.

It can be notice that the left part of this formulation can be developed in a sum of integrals whose number depends on the non-zero terms of $\mathbf{H}$.

\subsection{A model for shell structures}

\subsubsection{An adapted separated representation}

The idea is to use the PGD method separating a solid shell structure in two spaces: the mid-plane surface and the thickness. The shell structure occupies a region $\Omega=\mathcal{S} \times \mathcal{T}$ where $\mathcal{S}$ describes the mid-plane surface and $\mathcal{T}=\left[-\frac{e}{2} ; \frac{e}{2}\right]$ is an interval containing all the positions in the thickness defined by a signed distance from the mid-plane. $e$ denotes the thickness of the shell (or the maximum thickness in the case of a non constant thickness). In the following, the curvature is assumed to be the same in all the thickness. This assumption is adapted for shell with small thickness or law curvature. The global Cartesian coordinates are noted $\mathbf{X}=(X, Y, Z)$ with $\left(\mathbf{e}_{X}, \mathbf{e}_{Y}, \mathbf{e}_{Z}\right)$ the corresponding basis. A local basis $\left(\mathbf{e}_{x}, \mathbf{e}_{y}, \mathbf{e}_{z}\right)$ is also defined at each point of $\mathcal{S}$. We denote $\mathbf{x}=(x, y, z)$ the local coordinates where $x$ and $y$ are the coordinates along two directions parallel to the mid-plane surface and $z$ is the perpendicular direction. In local coordinate, the mid-place surface is defined by $z=0 . \mathbf{u}=(u, v, w)$ denotes the local displacement.

Then, a separated representation related to shell structures lies in:

$$
\left\{\begin{array}{l}
u(x, y, z) \approx \sum_{i=1}^{N} F_{i}^{u}(x, y) G_{i}^{u}(z) \\
v(x, y, z) \approx \sum_{i=1}^{N} F_{i}^{v}(x, y) G_{i}^{v}(z) \forall(x, y, z) \in \Omega \\
w(x, y, z) \approx \sum_{i=1}^{N} F_{i}^{w}(x, y) G_{i}^{w}(z)
\end{array}\right.
$$

The vector functions $\mathbf{F}_{i}=\left(F_{i}^{u}, F_{i}^{v}, F_{i}^{w}\right)$ are defined over the midplane surface $\mathcal{S}$ and the vector functions $\mathbf{G}_{i}=\left(G_{i}^{u}, G_{i}^{v}, G_{i}^{w}\right)$ are defined over the thickness of the shell structure (related to the local out-of-plane coordinate $z$ ). Practically, a shell or plate mesh is used to describe the mid-plane space and a simple $1 \mathrm{D}$ mesh is used to treat the out of plane space. The nodes of the shell mesh are generally defined in global coordinates and the shell elements are treated in local coordinates using a change of basis.

For sake of clarity, the PGD method will be described considering the weak form of Eq. (5) without volume force and acceleration and in the case of a linear isotropic homogeneous material. The shell structures will be considered as infinite along $\mathbf{e}_{y}$. A 2D plane strain model is assumed in the plan $\left(\mathbf{e}_{x}, \mathbf{e}_{z}\right)$. The local basis and the mid-plane surface can then be described in 2D (Fig. 1). 1D element can be used to describe the mid-plane surface $\mathcal{S}$.

All the following development can be generalized for more complicated 3D cases without theoretical difficulties. The 2D plane strain comportment law is in the local system:

$$
\left[\begin{array}{c}
\sigma_{x x} \\
\sigma_{z z} \\
\sigma_{x z}
\end{array}\right]=\underbrace{\left[\begin{array}{ccc}
H_{11} & H_{13} & 0 \\
H_{13} & H_{33} & 0 \\
0 & 0 & H_{55}
\end{array}\right]}_{\mathbf{H}}\left[\begin{array}{c}
\varepsilon_{x x} \\
\varepsilon_{z z} \\
2 \varepsilon_{x z}
\end{array}\right]
$$




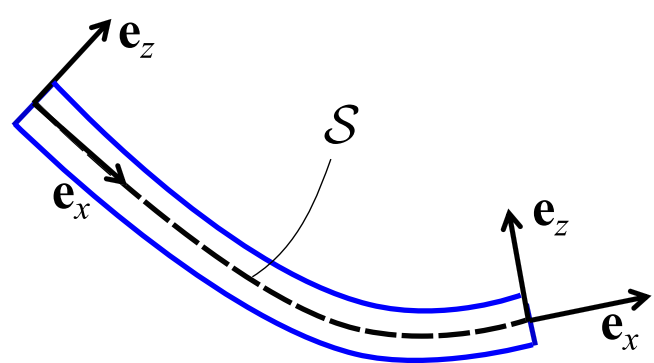

Fig. 1. Local basis definition.

$\mathbf{H}^{-\mathbf{1}}=\left[\begin{array}{ccc}\frac{1}{E_{x}} & -\frac{v_{x z}}{E_{x}} & 0 \\ -\frac{v_{x z}}{E_{x}} & \frac{1}{E_{z}} & 0 \\ 0 & 0 & \frac{1}{G_{x z}}\end{array}\right]$

Then the weak form becomes:

$$
\begin{aligned}
& \int_{\Omega} \varepsilon_{x x}^{\star}\left(H_{11} \varepsilon_{x x}+H_{13} \varepsilon_{z z}\right)+\int_{\Omega} \varepsilon_{z z}^{\star}\left(H_{13} \varepsilon_{x x}+H_{33} \varepsilon_{z z}\right)+\int_{\Omega} 2 \varepsilon_{x z}^{\star} H_{55} \varepsilon_{x z} \\
& =\int_{\partial \Omega} \mathbf{u}^{\star} \cdot(\boldsymbol{\sigma} \cdot \mathbf{n})
\end{aligned}
$$

and the PGD approximation reduced to:

$$
\left\{\begin{array}{l}
u(x, z) \approx \sum_{i=1}^{N} F_{i}^{u}(x) G_{i}^{u}(z) \\
w(x, z) \approx \sum_{i=1}^{N} F_{i}^{w}(x) G_{i}^{w}(z)
\end{array} \forall(x, z) \in \Omega\right.
$$

The expression of the gradient of displacement and then of the strain tensor $\boldsymbol{\varepsilon}$ in the local basis needs the use of the covariant derivative to account for the curvature over an element.

It can be noticed that the local basis depends only on the position in the mid-plane surface $x$. It does not evolve in the thickness so that $\frac{\partial \mathbf{e}_{x}}{\partial z}=\frac{\partial \mathbf{e}_{y}}{\partial z}=\frac{\partial \mathbf{e}_{z}}{\partial z}=0$.

With the thin-shell assumption, the strain tensor writes:

$\varepsilon_{x x}=\frac{\partial \mathbf{u}}{\partial x} \cdot \mathbf{e}_{x}=\frac{\partial}{\partial x}\left(u \mathbf{e}_{x}+w \mathbf{e}_{z}\right) \cdot \mathbf{e}_{x}=\frac{\partial u}{\partial x}+w\left(\frac{\partial \mathbf{e}_{z}}{\partial x} \cdot \mathbf{e}_{x}\right)$

and also:

$\varepsilon_{z z}=\frac{\partial \mathbf{u}}{\partial z} \cdot \mathbf{e}_{z}=\frac{\partial w}{\partial z}$

$2 \varepsilon_{x z}=\frac{\partial \mathbf{u}}{\partial z} \cdot \mathbf{e}_{x}+\frac{\partial \mathbf{u}}{\partial x} \cdot \mathbf{e}_{z}=\frac{\partial u}{\partial z}+\frac{\partial w}{\partial x}+u\left(\frac{\partial \mathbf{e}_{x}}{\partial x} \cdot \mathbf{e}_{z}\right)$

Remark 1. The expression of the strain tensor can be generalized for deep shells. However, in the case of deep shells, the separated formulation of the problem needed to use the PGD become more complicated and not very efficient in a computational point of view.

The terms $\frac{\partial \mathbf{e}_{x}}{\partial x}$ and $\frac{\partial \mathbf{e}_{z}}{\partial x}$ represent the evolution of the local basis. An interpolation of the local basis is defined on each element, and this interpolation allows to compute the evolution of the local basis. In practical, the local basis is defined at nodes and is interpolated over the element. The local basis can be set independently of the element geometry though it should reasonably be related to it. For instance, plate elements may be used to model a curved geometry even if there is no curvature over this kind of elements. The local basis varies and follows the curvature of the real geometry and is then continuous in the whole geometry. Therefore, elements with plate or shell geometry may be used indifferently.

We denote $f(x)=\left(\frac{\partial \mathbf{e}_{z}}{\partial x} \cdot \mathbf{e}_{x}\right)$ and $g(x)=\left(\frac{\partial \mathbf{e}_{x}}{\partial x} \cdot \mathbf{e}_{z}\right)$.

Considering Eqs. (11)-(13), the weak formulation writes:

$$
\begin{aligned}
& \int_{\Omega} H_{11}\left(\frac{\partial u^{\star}}{\partial x}+f w^{\star}\right)\left(\frac{\partial u}{\partial x}+f w\right)+\int_{\Omega} H_{33} \frac{\partial w^{\star}}{\partial z} \frac{\partial w}{\partial z} \\
& \quad+\int_{\Omega} H_{13}\left(\frac{\partial u^{\star}}{\partial x}+f w^{\star}\right) \frac{\partial w}{\partial z}+\int_{\Omega} H_{13} \frac{\partial w^{\star}}{\partial z}\left(\frac{\partial u}{\partial x}+f w\right) \\
& \quad+\int_{\Omega} \frac{H_{55}}{2}\left(\frac{\partial u^{\star}}{\partial z}+\frac{\partial w^{\star}}{\partial x}+g u^{\star}\right)\left(\frac{\partial u}{\partial z}+\frac{\partial w}{\partial x}+g u\right)=\int_{\partial \Omega} \mathbf{u}^{\star} \cdot(\boldsymbol{\sigma} \cdot \mathbf{n})
\end{aligned}
$$

The left part of this equation can be developed in 18 simple integrals.

\subsubsection{Enriching the approximation basis}

At the iteration $N$ the approximation is assumed known under the form given by Eq. (10). To enrich this approximation, 4 functions must be determined (6 in the general 3D case): $F_{N+1}^{u}, G_{N+1}^{u}, F_{N+1}^{w}$ and $G_{N+1}^{w}$ (or $\mathbf{F}_{N+1}$ and $\mathbf{G}_{N+1}$ if we use vector functions).

$$
\left\{\begin{array}{l}
u(x, z) \approx \sum_{i=1}^{N} F_{i}^{u}(x) G_{i}^{u}(z)+F_{N+1}^{u}(x) G_{N+1}^{u}(z) \\
w(x, z) \approx \sum_{i=1}^{N} F_{i}^{w}(x) G_{i}^{w}(z)+F_{N+1}^{w}(x) G_{N+1}^{w}(z)
\end{array} \forall(x, z) \in \Omega\right.
$$

Remark 2. In the first iteration step, no function is known. $\mathbf{F}_{1}$ and $\mathbf{G}_{1}$ have then to be determined.

To determine $\mathbf{F}_{N+1}$ and $\mathbf{G}_{N+1}$ a non-linear problem has to be solved. An alternate directions strategy has given excellent results in our precedent studies (for example in [15]). This method is performed in two steps:

- Step 1: $\mathbf{G}_{N+1}$ being known, we are looking for $\mathbf{F}_{N+1}$.

- Step 2: $\mathbf{F}_{N+1}$ being known, we are looking for $\mathbf{G}_{N+1}$.

Starting with an arbitrary tentative functions $\mathbf{G}_{N+1}$, step 1 is performed and then step 2, and again both steps until reaching convergence. The convergence is obtained when the norms of the difference between the two last values of $\mathbf{F}_{N+1}$ and $\mathbf{G}_{N+1}$ are sufficiently small.

Step 1. In step $1, \mathbf{G}_{N+1}$ is assumed known. So the trial function $\mathbf{u}^{\text {tr }}$ is given by

$$
\left\{\begin{array}{l}
u^{\star}(x, z)=F_{N+1}^{u} \star(x) G_{N+1}^{u}(z) \\
w^{\star}(x, z)=F_{N+1}^{w} \star(x) G_{N+1}^{w}(z)
\end{array} \forall(x, z) \in \Omega\right.
$$

The function $\mathbf{G}_{\mathbf{N}+\mathbf{1}}$ depends only on the position $z$ along the thickness and the function $\mathbf{F}_{\mathbf{N}+\mathbf{1}}$ depends only on the position in the mid-plane surface.

With the thin-shell assumption, the derivative with respect to the local coordinate $x$ does not depend on the position in the thickness and the integrals can be separated.

Then, we define the function $A$ such as: 


$$
\begin{aligned}
& A\left(\mathbf{F}_{i}^{\star}, \mathbf{F}_{j}, \mathbf{G}_{i}, \mathbf{G}_{j}\right)=\left[\int_{\mathcal{S}} H_{11} \frac{d F_{i}^{u \star}}{d x} \frac{d F_{j}^{u}}{d x}+\frac{H_{55}}{2} g^{2} F_{i}^{u \star} F_{j}^{u}\right] \times\left[\int_{\Omega_{z}} G_{i}^{u} G_{j}^{u}\right] \\
& +H_{33}\left[\int_{\mathcal{S}} F_{i}^{w \star} F_{j}^{w}\right] \times\left[\int_{\Omega_{z}} \frac{d G_{i}^{w}}{d z} \frac{d G_{j}^{w}}{d z}\right] \\
& +H_{13}\left[\int_{\mathcal{S}} F_{i}^{w \star} \frac{d F_{j}^{u}}{d x}\right] \times\left[\int_{\Omega_{z}} \frac{d G_{i}^{w}}{d z} G_{j}^{u}\right] \\
& +H_{13}\left[\int_{\mathcal{S}} \frac{d F_{i}^{u \star}}{d x} F_{j}^{w}\right] \times\left[\int_{\Omega_{z}} G_{i}^{u} \frac{d G_{j}^{w}}{d z}\right] \\
& +\frac{H_{55}}{2}\left[\int_{\mathcal{S}} F_{i}^{u \star} F_{j}^{u}\right] \times\left[\int_{\Omega_{z}} \frac{d G_{i}^{u}}{d z} \frac{d G_{j}^{u}}{d z}\right] \\
& +\frac{H_{55}}{2}\left[\int_{\mathcal{S}} F_{i}^{u \star} \frac{d F_{j}^{w}}{d x}\right]\left[\int_{\Omega_{z}}\left(\frac{d G_{i}^{u}}{d z} G_{j}^{w}\right)\right] \\
& +\frac{H_{55}}{2}\left[\int_{\mathcal{S}} \frac{d F_{i}^{w \star}}{d x} F_{j}^{u}\right]\left[\int_{\Omega_{z}}\left(G_{i}^{w} \frac{d G_{j}^{u}}{d z}\right)\right] \\
& +\left[\int_{\mathcal{S}} \frac{H_{55}}{2} \frac{d F_{i}^{w \star}}{d x} \frac{d F_{j}^{w}}{d x}+H_{11} f^{2}\left(F_{i}^{w \star} F_{j}^{w}\right)\right] \times\left[\int_{\Omega_{z}} G_{i}^{w} G_{j}^{w}\right] \\
& +\left[\int_{\mathcal{S}} H_{11} f \frac{d F_{i}^{u \star}}{d x} F_{j}^{w}+\frac{H_{55}}{2} g F_{i}^{u \star} \frac{d F_{j}^{w}}{d x}\right] \times\left[\int_{\Omega_{z}} G_{i}^{u} G_{j}^{w}\right] \\
& \left.\left.+\left[\int_{\mathcal{S}} H_{11} f \quad F_{i}^{w \star} \frac{d F_{j}^{u}}{d x}\right)+\frac{H_{55}}{2} g \frac{d F_{i}^{w \star}}{d x} F_{j}^{u}\right)\right] \\
& \times\left[\int_{\Omega_{z}} G_{i}^{w} G_{j}^{u}\right]+H_{13}\left[\int_{\mathcal{S}} f F_{i}^{w \star} F_{j}^{w}\right] \\
& \times\left[\int_{\Omega_{z}} G_{i}^{w} \frac{d G_{J}^{w}}{d z}+\frac{d G_{i}^{w}}{d z} G_{j}^{w}\right] \\
& +\frac{H_{55}}{2}\left[\int_{\mathcal{S}} g F_{i}^{u \star} F_{j}^{u}\right]\left[\int_{\Omega_{z}} \frac{d G_{i}^{u}}{d z} G_{j}^{u}+G_{i}^{u} \frac{d G_{j}^{u}}{d z}\right]
\end{aligned}
$$

With this function, the weak form using the separated representation reads:

$$
\begin{aligned}
A\left(\mathbf{F}_{N+1}^{\star}, \mathbf{F}_{N+1}, \mathbf{G}_{N+1}, \mathbf{G}_{N+1}\right)= & \int_{\partial \Omega} \mathbf{u}^{\star} \cdot(\boldsymbol{\sigma} \cdot \mathbf{n}) \\
& -\sum_{i=1}^{N} A\left(\mathbf{F}_{N+1}^{\star}, \mathbf{F}_{i}, \mathbf{G}_{N+1}, \mathbf{G}_{i}\right)
\end{aligned}
$$

In the right part of this equation, every terms are known. In Eq. (17), the integrals over the thickness can be approximated numerically because the functions $\mathbf{G}_{i}$ are known $\forall i$. It remains only a problem defined over the mid-surface space. It reduces significantly the complexity of the problem. In practical, a finite element approximation is used over each sub domain.

Step 2. In step 2, $\mathbf{F}_{N+1}$ is assumed known. So the trial function $\mathbf{u}^{\star}$ is given by

$$
\left\{\begin{array}{l}
u^{\star}(x, z)=F_{N+1}^{u}(x) G_{N+1}^{u \star}(z) \\
w^{\star}(x, z)=F_{N+1}^{w}(x) G_{N+1}^{w \star}(z)
\end{array} \forall(x, z) \in \Omega\right.
$$

Now, the weak form using the separated representation becomes:

$A\left(\mathbf{F}_{N+1}, \mathbf{F}_{N+1}, \mathbf{G}_{N+1}^{\star}, \mathbf{G}_{N+1}\right)=\int_{\partial \Omega} \mathbf{u}^{\star} \cdot(\boldsymbol{\sigma} \cdot \mathbf{n})-\sum_{i=1}^{N} A\left(\mathbf{F}_{N+1}, \mathbf{F}_{i}, \mathbf{G}_{N+1}^{\star}, \mathbf{G}_{i}\right)$

The integrals over $\mathcal{S}$ can be approximated numerically because the functions $\mathbf{F}_{i}$ are known $\forall i$. It remains only a problem defined over $\mathcal{T}$ which can be solved using a finite element solver.
The convergence criterion is based on the norm of the residual. The residual is computed from the Finite Element operators after discretization on each subspace. See [8] for more detail on this criterion.

Remark 3. A projection step helps sometimes to improve the convergence of the algorithm as proposed in [1]. This step is not compulsory though.

\section{Results}

\subsection{Validation of the method}

A simple numerical test is performed to test the accuracy of the method. It consists on a laminated cylindrical shells in cylindrical bending. The geometry is described Fig. 2. The exact solution is given by J.G. Ren in [18]. The shell is considered as infinite along the $z$ axis so that a plane strain assumption is used. The shell is simply supported on its edges and the upper surface is loaded with a normal traction $\sigma_{r}=q(\theta)$ with $q(\theta)=q_{0} \sin (3 \theta)$.

The boundary conditions on the edges are:

$w(r, 0)=w\left(r, \frac{\pi}{3}\right)=0$

Here $w(r, \theta)$ denotes the displacement in the $r$-direction (along $\mathbf{e}_{z}$ in the shell local basis) and $u(r, \theta)$ denotes the displacement in the $\theta$ direction (along $\mathbf{e}_{x}$ in the shell local basis).

To suppress rigid body motions, another condition may be added:

$u\left(r, \frac{\pi}{6}\right)=0$

This condition is not compulsory to make the PGD converges but if it is omitted there is an infinite number of possible solutions and only one will be obtained by the fixed point algorithm used in the PGD solver.

For the test case, a symmetric [0/90/0] CFRP laminate with unidirectional plies is considered. The materials properties are the ones used in [18]:

$$
\begin{array}{ll}
E_{L}=172 \mathrm{GPa} & E_{T}=6.9 \mathrm{GPa} \\
G_{L T}=3.4 \mathrm{GPa} & G_{T T}=1.4 \mathrm{GPa} \\
v_{L T}=v_{T T}=0.25 &
\end{array}
$$

$L$ is the direction parallel to the fibers and $T$ is the transverse direction.

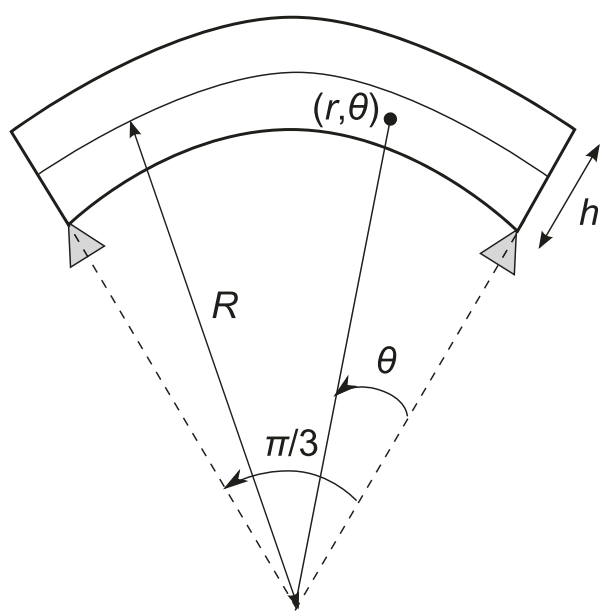

Fig. 2. Laminated cylindrical shell. 
Table 1

Results of the PGD compared with the exact solution for stresses and displacement.

\begin{tabular}{lllllll}
\hline $\begin{array}{l}S \\
(z, \theta)\end{array}$ & $\begin{array}{l}\bar{\sigma}_{x x} \\
\left(-\frac{h}{2}, \frac{\pi}{6}\right)\end{array}$ & $\begin{array}{l}\bar{\sigma}_{x x} \\
\left(\frac{h}{2}, \frac{\pi}{6}\right)\end{array}$ & $\begin{array}{l}\bar{\sigma}_{z z} \\
\left(\mathbf{0}, \frac{\pi}{6}\right)\end{array}$ & $\begin{array}{l}\bar{\sigma}_{x z} \\
(0,0)\end{array}$ & $\begin{array}{l}\bar{u} \\
\left(-\frac{h}{2}, 0\right)\end{array}$ & $\begin{array}{l}\bar{w} \\
\left(0, \frac{\pi}{6}\right)\end{array}$ \\
\hline Exact & & & & & & \\
4 & -1.761 & 1.36 & -0.00103 & 0.478 & 8.217 & 0.457 \\
10 & -0.993 & 0.895 & -0.0121 & 0.526 & 6.063 & 0.144 \\
50 & -0.798 & 0.783 & -0.0033 & 0.525 & 14.56 & 0.081 \\
100 & -0.786 & 0.779 & -0.00169 & 0.523 & 27.31 & 0.0788 \\
$P G D$ & & & & & & \\
4 & -1.37 & 1.39 & -0.0041 & 0.429 & 7.368 & 0.411 \\
10 & -0.904 & 0.893 & -0.0117 & 0.501 & 5.792 & 0.137 \\
50 & -0.785 & 0.781 & -0.00328 & 0.521 & 14.42 & 0.0802 \\
100 & -0.781 & 0.777 & -0.0017 & 0.524 & 27.18 & 0.0784 \\
\hline
\end{tabular}

The results are obtained with the PGD using only 1D quadratic elements for the mid-plane surface and for the thickness. For this test case, there is 100 elements in the mid-plane and surface and 60 elements in the thickness. In Table 1 the displacement and stress are normalized by:

$\left(\bar{\sigma}_{x x}, \bar{\sigma}_{z z}\right)=\frac{1}{q_{0} S^{2}}\left(\sigma_{x x}, \sigma_{z z}\right) \quad \bar{\sigma}_{x z}=\frac{1}{q_{0} S} \sigma_{x z} \quad \bar{u}=\frac{100 E_{T} u}{q_{0} h S^{3}} \quad \bar{w}=\frac{10 E_{T} w}{q_{0} h S^{4}}$

where $S=R / h$. A normalized $z$-position is also used in the display: $\bar{z}=z / h$.

The Table 1 shows a rather good agreement between the PGD and the exact solution. No shear and membrane locking can be observed (even if linear elements are used instead of quadratic elements). The error decreases when $S$ increases. This can be explained with the assumption that the curvature is the same in the thickness (small thickness assumption). The error on maximal stresses and displacements is about $10 \%$ for $S=4$ and $0.3 \%$ for $S=100$. Figs. 3 and 4 shows the good agreement between the PGD method and the exact solution for $S=50$. For this problem the convergence of the PGD is obtained with only 2 or 3 enrichment iterations.

The presented method gives reliable results in the case of reasonably thin thickness shell. For value of $S$ higher than 10 the results are acceptable. The stress field obtained for $S=10$ and the error with the exact solution is depicted in Fig. 5.

\subsection{A 3D test case}

The results obtained by the PGD for a full 3D test case are compared with the ones obtained by the FEM using 3D solid elements. This test case is a square panel of $100 \mathrm{~mm}$ side length and $5 \mathrm{~mm}$ thickness. This panel is curved with different curvatures along the two axis. The geometry and the nodal local basis are depicted Fig. 6. The edges of the panel are clamped and a normal force of $100 \mathrm{kN}$ is applied at the center of the upper face (a concentrated force in the sense of the finite element approximation which is in reality distributed over the adjacent elements). To ease the comparison with the FEM an isotropic linear elastic constitutive law is used ( $E=70 \mathrm{GPa}$ and $v=0.3$ ) and the domain is homogeneous.

The deformed geometry (with a displacement increased for sake of visibility) is depicted in Fig. 7. This figure shows also that the error on the displacement between the reduced approach (PGD) and the full FEM simulation is relatively small all over the panel. The mean relative error on displacement is $0.58 \%$ and $3 \%$ on each component of the stress field. The profile of $\sigma_{x x}$ at the center of the panel is depicted for both methods in Fig. 8. The two methods give similar results. Here again, the difference can be explained by the thin shell assumption used for the PGD and also by the difference of interpolation function between the PGD and
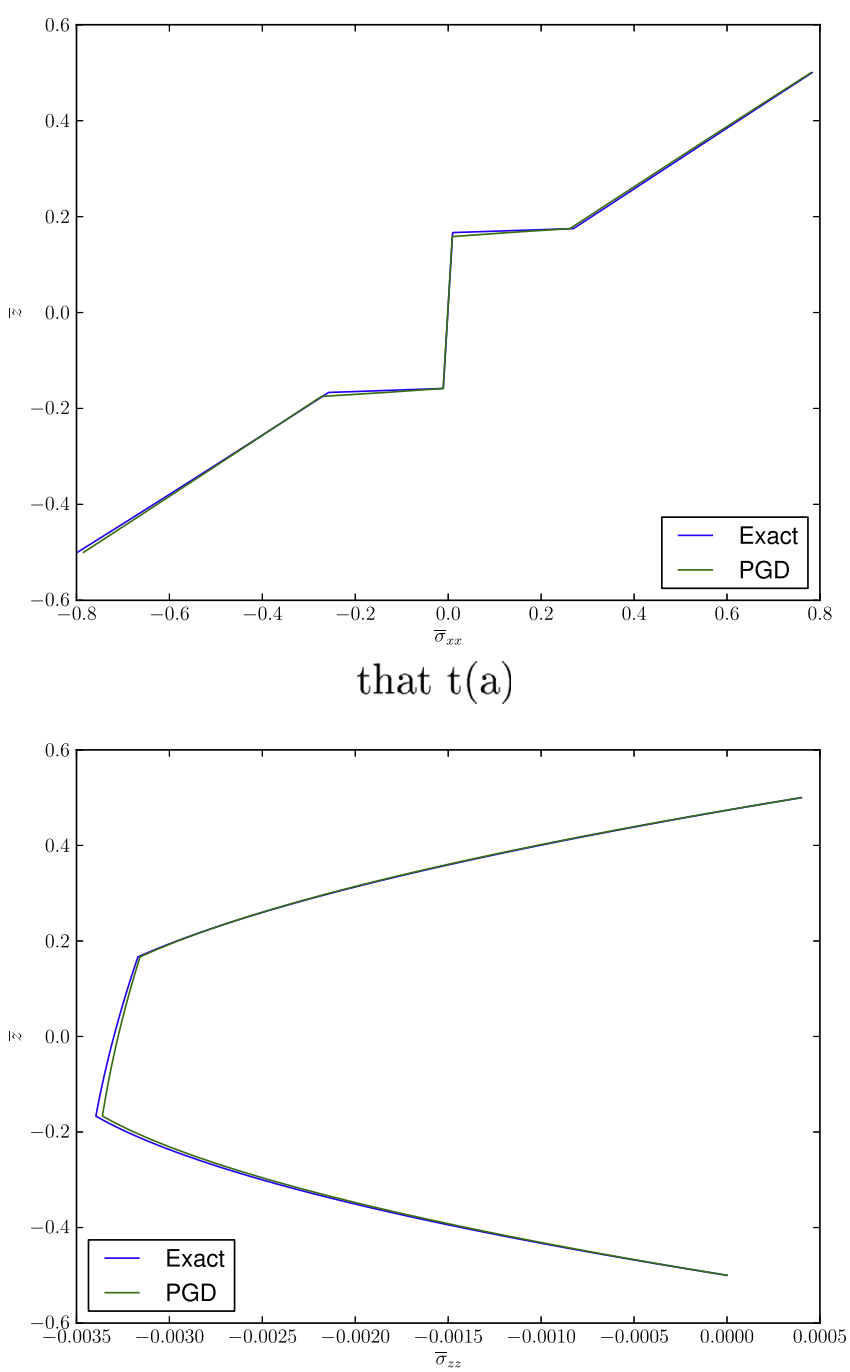

(b)

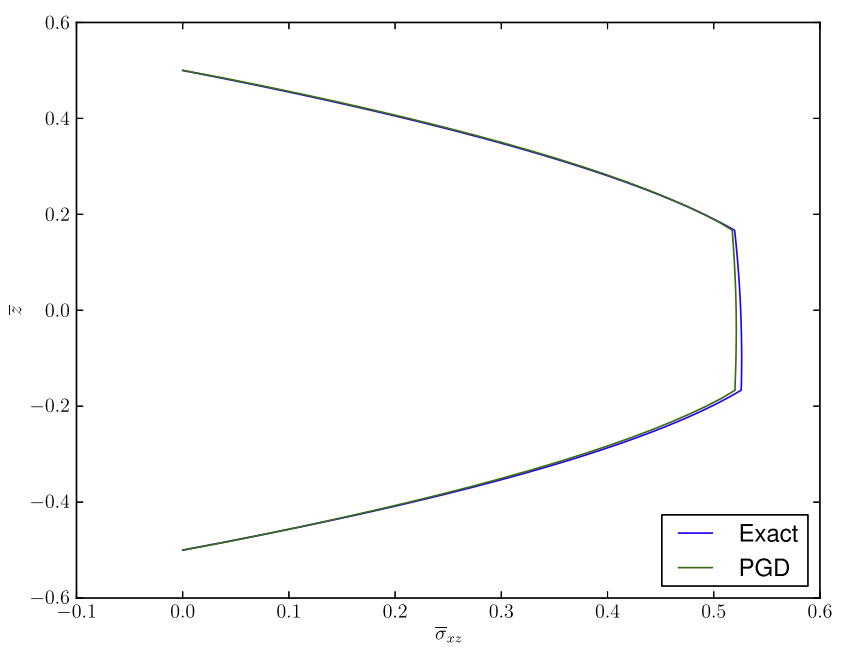

(c)

Fig. 3. Stress for $S=50$ : (a) $\bar{\sigma}_{x x}$ over the thickness for $\theta=\frac{\pi}{6}$. (b) $\bar{\sigma}_{z z}$ over the thickness for $\theta=\frac{\pi}{6}$. (c) $\bar{\sigma}_{x z}$ over the thickness for $\theta=0$.

the finite element method. It can be noticed that the longitudinal stress is mainly compressive because of the curvature of the shell (the model does not account for large displacements). 


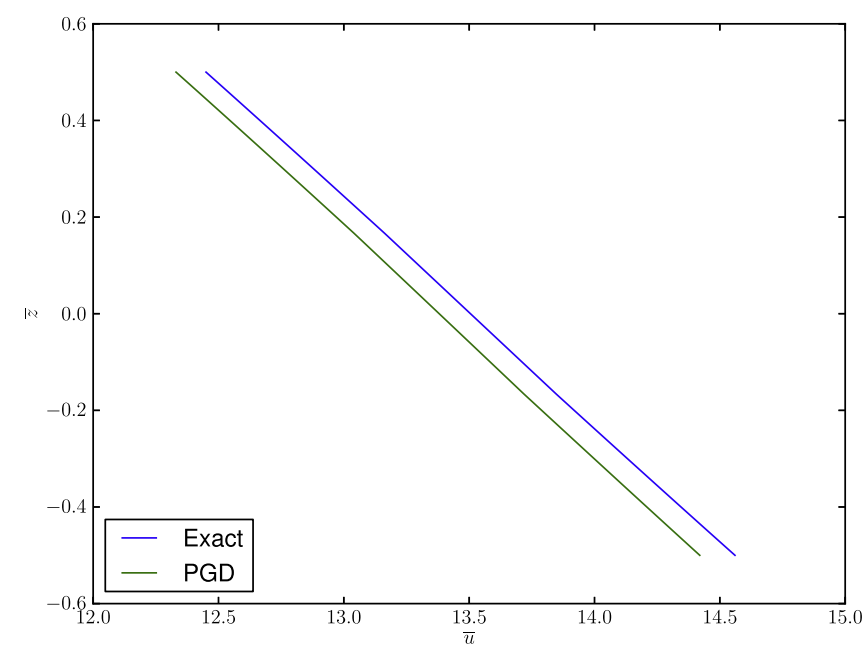

Fig. 4. Displacement $\bar{u}$ over the thickness for $\theta=0$ and $S=50$.

\section{A problem dependent shell approximation}

\subsection{Strategy}

As already said, the main advantage of the PGD is that it is based on the full 3D model with a 2D complexity. However, shell models are generally more efficient in a computational point of view because they require only one $2 \mathrm{D}$ calculation when the PGD requires many 2D calculation (with less degrees of freedom though).
In this section, a global strategy is proposed to combine the advantages of the PGD and the advantages of shell elements.

The PGD algorithm builds the solution on a separated form given in Eq. (6). This separated approximation is close to the Carrera Unified Formulation [5] from which most of shell models can be derived. In the Carrera Unified Formulation, the functions related to the thickness depend on the chosen model and are defined prior to the calculation.

The PGD algorithm presented in this paper can be used to compute these basis functions. Actually, the PGD can be used on a given problem to determine a set of functions $\mathbf{G}_{i}$ related to the thickness. Once these functions are known, they can be used as basis functions on a different (but close enough) problem. In general, there is no evidence that a set of functions computed for a given problem will be adapted to treat another problem. But in the context of shell structures, the functions related to the thickness have physical sense. They represent a combination of modes related to bending, rigid displacements, compression of the normal fiber and also more complex modes related to boundary effects. Of course, the use of a basis has no sense if the laminate is not the same. In particular, this strategy can't be used to optimize a laminate. An appropriate basis is build with a former problem (or with many former problems) including all the solicitations that are required in the model.

A set of functions $\mathbf{G}_{i}$ with $i=\{1,2, \ldots, N\}$ is assumed known from a previous problem. It remains to determine the set of associated functions related to the mid-plane surface $\mathbf{F}_{i}$. Then the virtual field writes:

$$
\left\{\begin{array}{l}
u^{\star}(x, z)=\sum_{i=1}^{N} F_{i}^{u \star}(x) G_{i}^{u}(z) \\
w^{\star}(x, z)=\sum_{i=1}^{N} F_{i}^{w \star}(x) G_{i}^{w}(z)
\end{array} \forall(x, z) \in \Omega\right.
$$
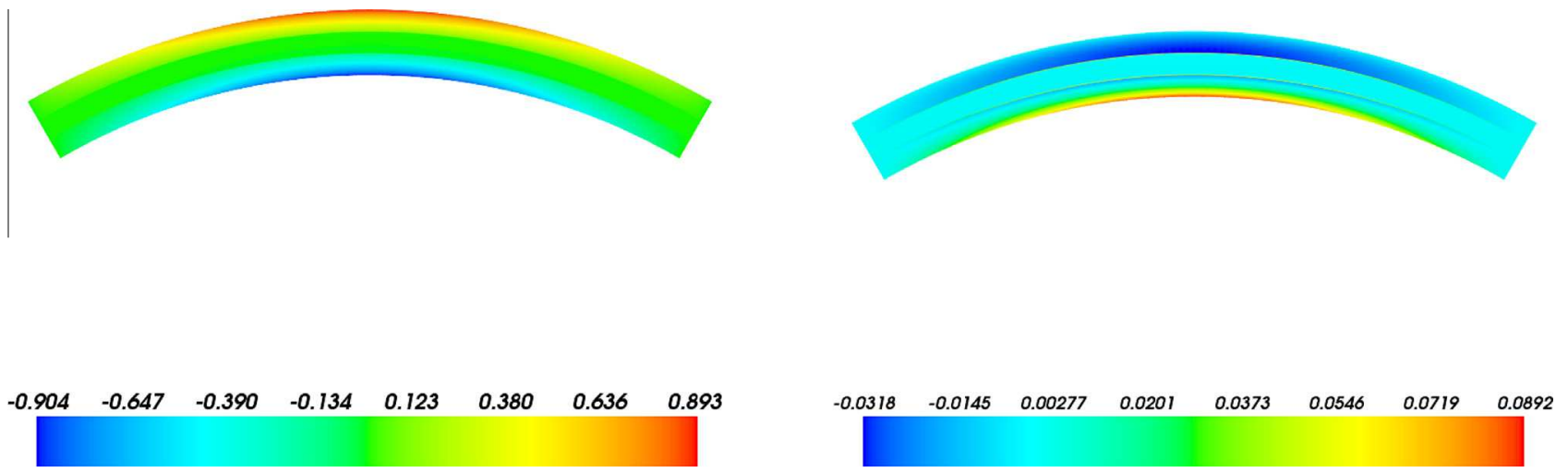

Fig. 5. $\bar{\sigma}_{x x}$ field obtained with the PGD for $S=10$ (left) - Error in comparison with the exact solution for $S=10$ (right).
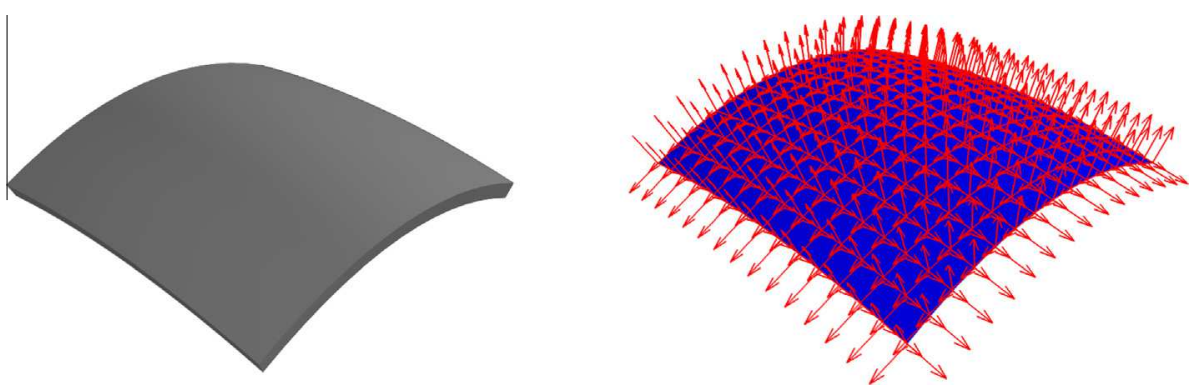

Fig. 6. Curved geometry (left). Mid-plane surface and local basis at nodes (right). 

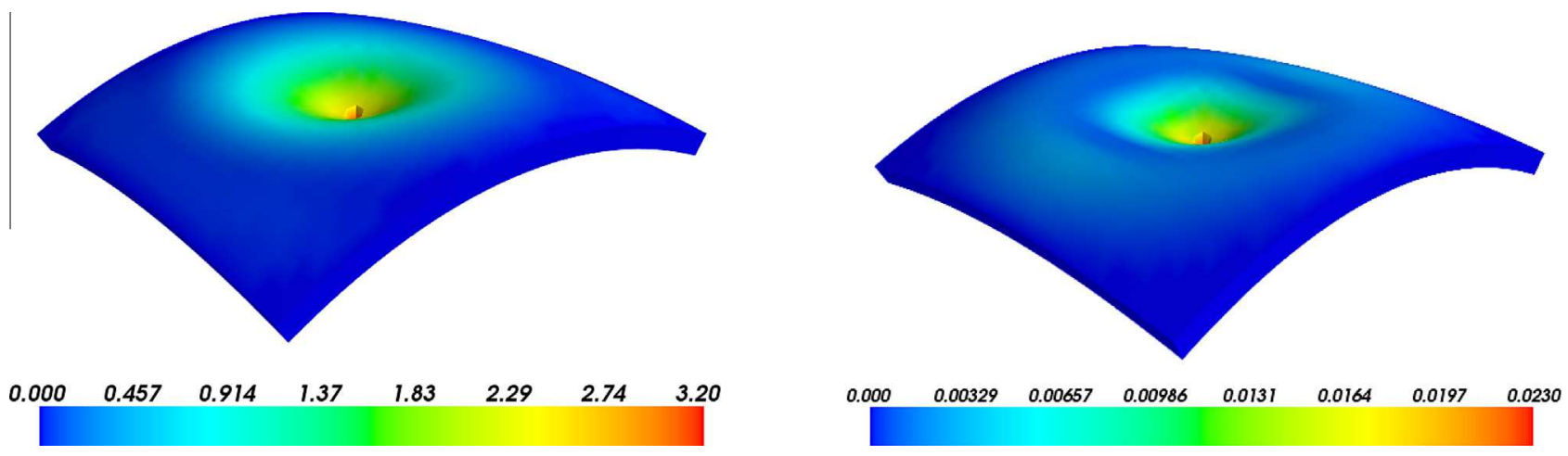

Fig. 7. Left: deformed shape and norm of displacement ( $\mathrm{mm})$. Right: error of the displacement between FEM and PGD (mm).

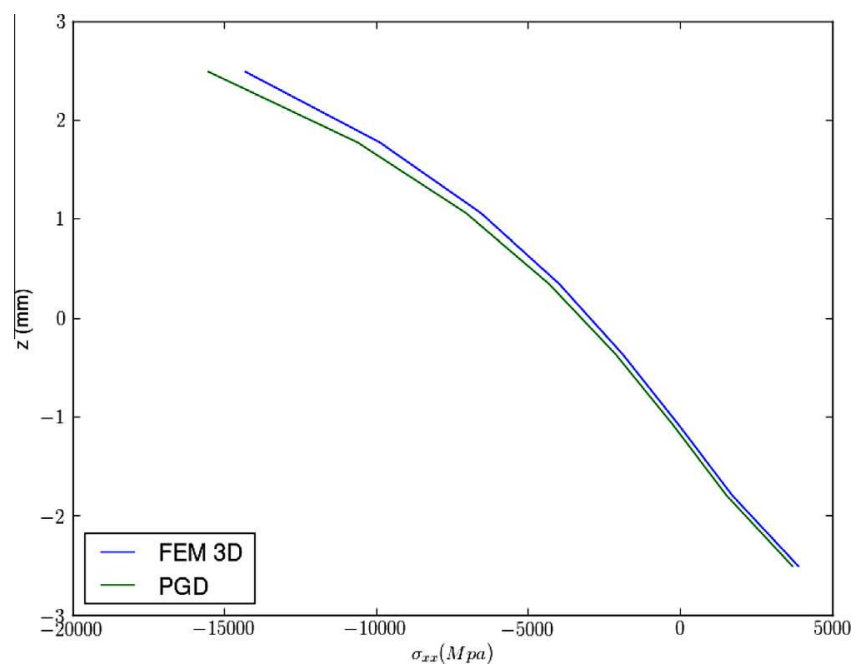

Fig. 8. $\sigma_{x x}$ over the thickness at the center of the panel.

And the displacement is approximated by:

$$
\left\{\begin{array}{l}
u(x, z)=\sum_{i=1}^{N} F_{i}^{u}(x) G_{i}^{u}(z) \\
w(x, z)=\sum F_{i}^{w}(x) G_{i}^{w}(z)
\end{array} \forall(x, z) \in \Omega\right.
$$

With the weak formulation Eq. (14) and using the function $A$ defined in Eq. (17) it finally gives:

$$
\sum_{i, j=1}^{N} A\left(\mathbf{F}_{i}^{\star}, \mathbf{F}_{j}, \mathbf{G}_{i}, \mathbf{G}_{j}\right)=\int_{\partial \Omega} \mathbf{u}^{\star} \cdot(\boldsymbol{\sigma} \cdot \mathbf{n})
$$

This system can be solved using a finite elements discretization, the unknown being the nodal values of all the functions $\mathbf{F}_{i}$ for $i=\{1,2, \ldots, N\}$.

Once these functions are determined, it is possible to compute the residual error related to the finite element operators (see [8] for more details). The error criterion used for the standard PGD can be applied here. If the error is not satisfying, new terms can be added with the standard PGD algorithm in order to converge toward a better solution. Then, it is easy to improve the basis by adding the new $z$-functions and making a Singular Value Decomposition.

The computational cost of the proposed strategy when the functions $\mathbf{G}_{i}$ are known should be in the same order as Layerwise approaches if the number of DOF is the same. The total number of DOF is related to the number $N$ of basis functions considered. The computational cost increases drastically when $N$ increases.
Therefore, the proposed strategy remains efficient since $N$ is sufficiently small. The initial PGD strategy mat be a better choice if the numbers of basis functions is to high (higher than 10 for instance). In addition, if the number of basis functions becomes higher than the total amount of nodes in the thickness, a 3D solid approach is probably more appropriate.

\subsection{Illustration}

To illustrate the above strategy, a new problem is firstly considered. It consists in a 2D shell (shell model using plane strain assumption) with uniform curvature. The mid-plane surface is described with a quarter of circle whose radius is set to $100 \mathrm{~mm}$. The thickness of the shell is set to $10 \mathrm{~mm}$. The shell is clamped on the left side and a $20 \mathrm{~mm}$ radial displacement is enforced on the right side.

A [0/90/0] laminate with unidirectional plies is considered with the properties given in Eq. (23).

The displacement of the shell is depicted in Fig. 9.

The results are obtained with the PGD using only 1D quadratic elements (see Fig. 10).

For this case, the PGD converges with 19 enrichment iterations. A Singular Value Decomposition of the solution gives an optimized decomposition with only 4 terms.

Now another problem based on an equivalent geometry is treated but with a different curvature (the radius of curvature is set to $200 \mathrm{~mm}$ ) and with a different boundary condition: a $20 \mathrm{~mm}$ tangential displacement is enforced on the right side. The displacement is depicted (Fig. 11). Finally the problem to solve is really

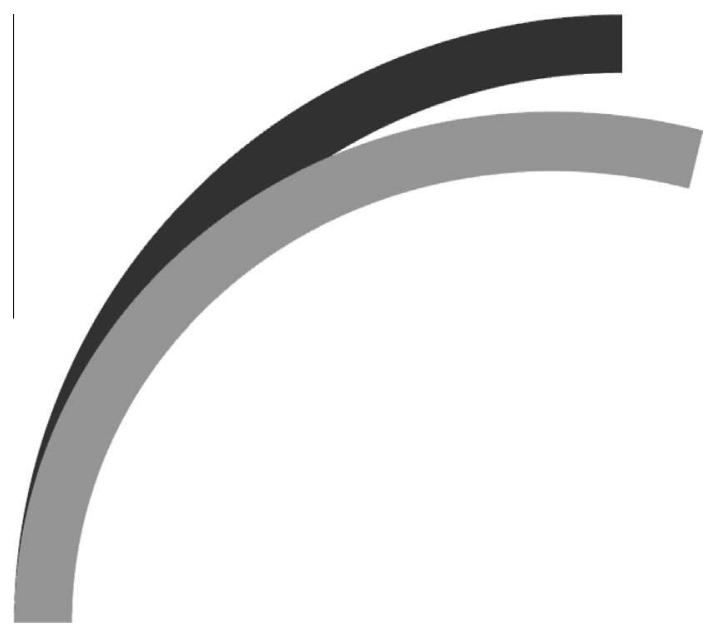

Fig. 9. Initial shape (dark grey) and deformed shape (light grey). 


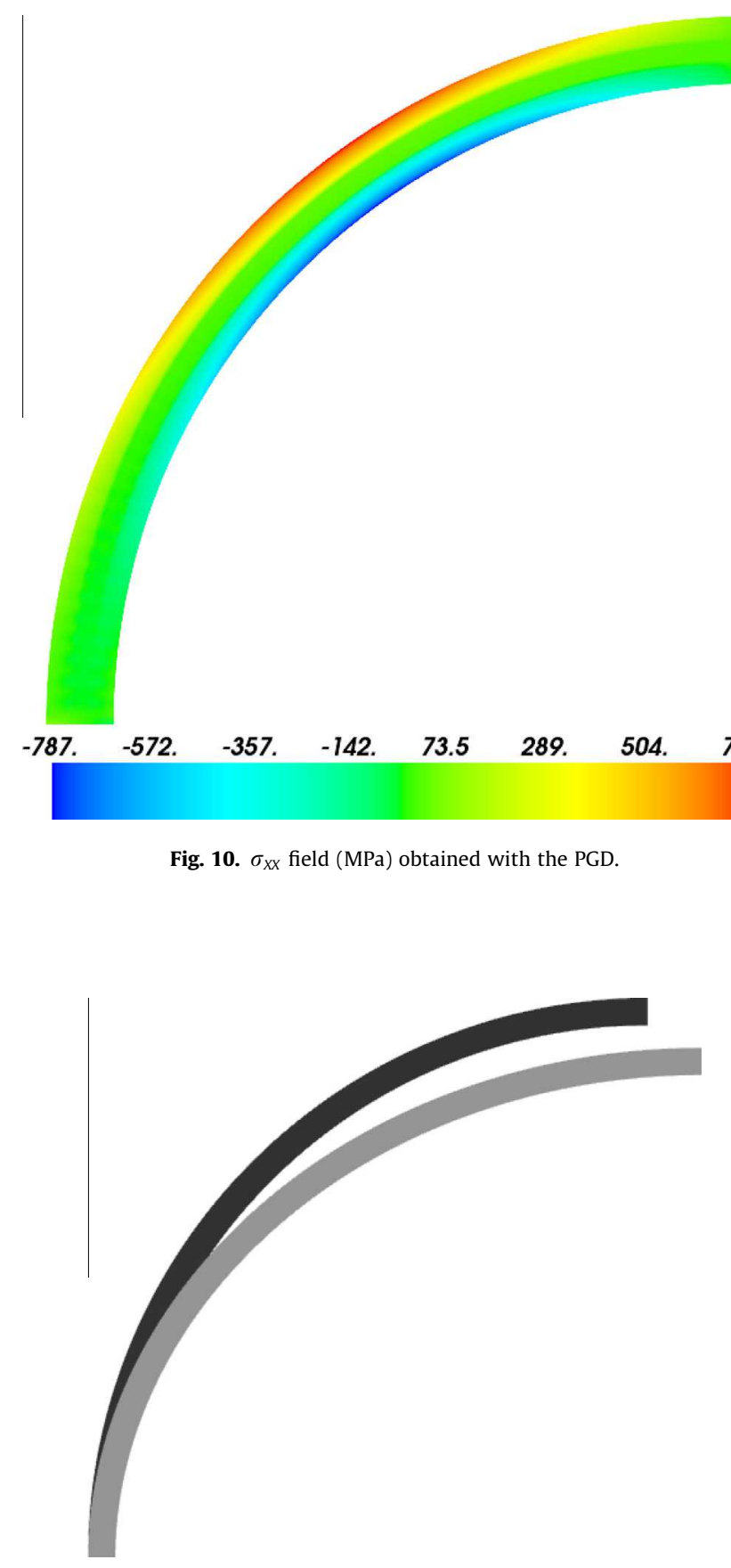

Fig. 11. Initial shape (dark grey) and deformed shape (light grey).

different from the previous one. This time, the solution is not determined with a new PGD algorithm but the functions $\mathbf{G}_{i}$ for $i=\{1,2,3,4\}$ are taken from the first calculus. Then, it remains to solve the $2 \mathrm{D}$ problem related to find $\mathbf{F}_{i}$ for $i=\{1,2,3,4\}$.

The results are compared with the solution obtained with the PGD method. The stress profiles are given in Fig. 12 on the most critical point that is on the bottom left of the structure for $\sigma_{x x}$ and $\sigma_{z z}$, and on the right extremity of the structure for $\sigma_{x z}$. The mean relative error (given as global indicator) is $0.08 \%$ for the displacement and around 3\% for the different stress components. Of course, locally, the error is more important as it can be seen in Fig. 12.

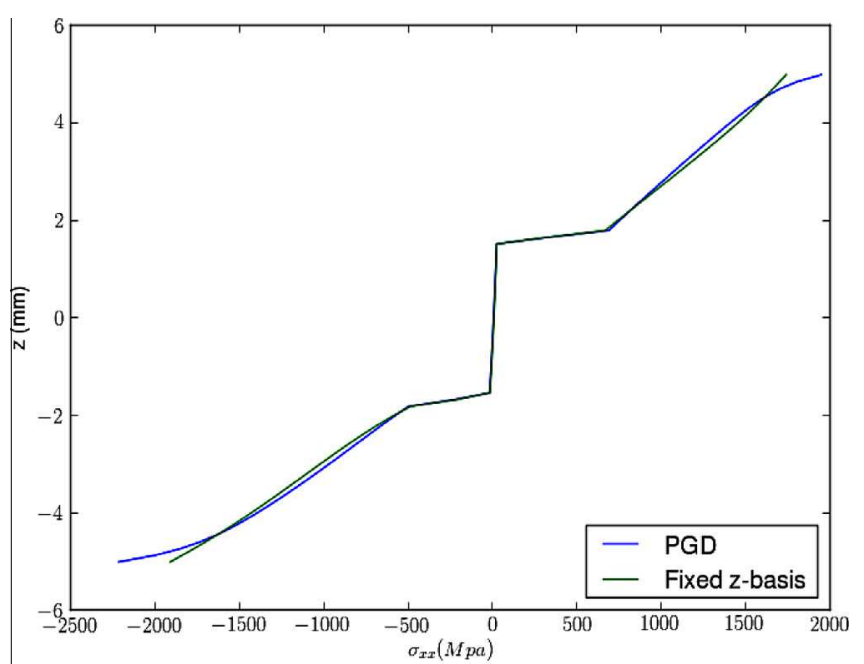

(a)

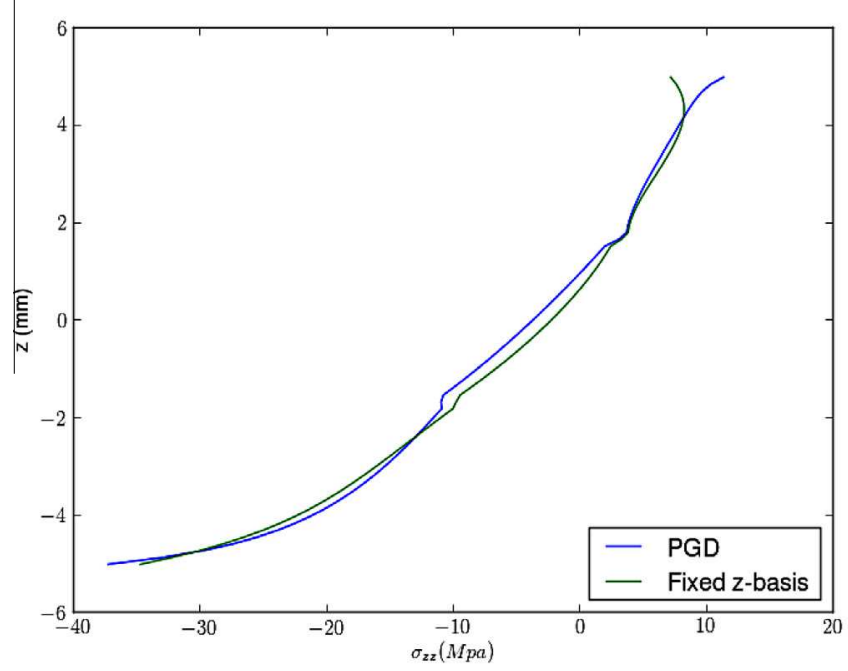

(b)

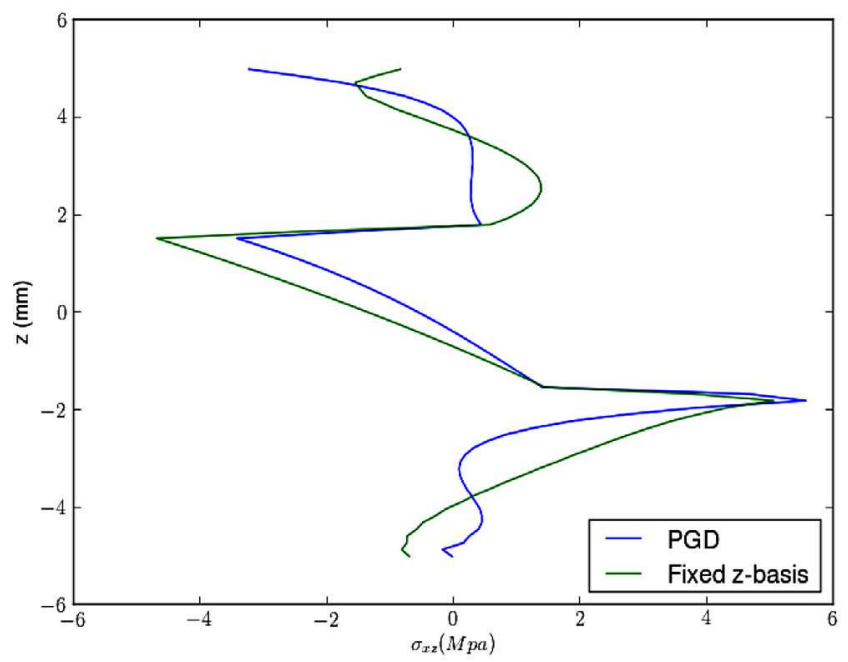

(c)

Fig. 12. Stress profile: (a) $\sigma_{x x}$ over the thickness on the bottom left of the structure (b) $\sigma_{z z}$ over the thickness on the bottom left of the structure. (c) $\sigma_{x z}$ over the thickness on the right of the structure. 


\section{Conclusion}

This article presents a new approach to treat shell structures between the use of shell elements and the use of 3D solid elements. The main interest of this approach is that it can treat complex phenomena like damaging in composites structures that are difficult to model with shell elements. Another advantage is that the computational cost is keep much lower than a full 3D finite elements modeling. Further work needs to be performed but this method is promising in the sense that it may bring in future significant advances in composites simulation.

\section{References}

[1] Ammar A, Mokdad B, Chinesta F, Keunings R. A new family of solvers for some classes of multidimensional partial differential equations encountered in kinetic theory modeling of complex fluids. J. Non-Newtonian Fluid Mech. 2006;139(3):153-76.

[2] Ammar A, Mokdad B, Chinesta F, Keunings R. A new family of solvers for some classes of multidimensional partial differential equations encountered in kinetic theory modeling of complex fluids. Part II: transient simulation using space-time separated representation. J. Non-Newtonian Fluid Mech. 2007; 144(2-3):98-121.

[3] Bognet B, Bordeu F, Chinesta F, Leygue A, Poitou A. Advanced simulation of models defined in plate geometries: 3D solutions with 2D computational complexity. Comput. Meth. Appl. Mech. Eng. 2012;201-204:1-12.

[4] Carrera E. Historical review of zig-zag theories for multilayered plates and shells. Appl. Mech. Rev. 2003:56(3):287-308.

[5] Carrera E, Ciuffreda A. A unified formulation to assess theories of multilayered plates for various bending problems. Compos. Struct. 2005;69(3):271-93.

[6] Chevreuil M, Nouy A. Model order reduction based on proper generalized decomposition for the propagation of uncertainties in structural dynamics. Int J. Numer. Methods Eng. 2012;89:241-68.
[7] Chinesta F, Ammar A, Lemarchand F, Beauchene P, Boust F. Alleviating mesh constraints: model reduction, parallel time integration and high resolution homogenization. Comput. Meth. Appl. Mech. Eng. 2008;197(5):400-13.

[8] Pruliere E, Chinesta F, Ammar A. On the deterministic solution of multidimensional parametric models using the proper generalized decomposition. Math. Comput. Simul. 2010;81(4):791-810.

[9] Gonzalez D, Ammar A, Chinesta F, Cueto E. Recent advances on the use of separated representations. Int. J. Numer. Methods Eng. 2010;81(5):637-59.

[10] P. Ladevèze, New algorithms: mechanical framework and development. Technical Report 57 (in French).

[11] Ladevèze P. On a family of algorithms for structural mechanics. CR Acad. Sci. Paris 1985:300(2):41-4 (in French).

[12] J.L. Lumley, The structure of inhomogeneous turbulent flows, 1967.

[13] Mokdad B, Prulière E, Ammar A, Chinesta F. On the simulation of kinetic theory models of complex fluids using the Fokker-Planck approach. Appl. Rheol. $2007 ; 17(2)$.

[14] Nouy A, Chevreuil M, Safatly E. Proper generalized decomposition for the solution of boundary value problems on uncertain parameterized domains. Comput. Meth. Appl. Mech. Eng. 2011;200(45-46):3066-82.

[15] Prulière E, Ammar A, El Kissi N, Chinesta F. Recirculating flows involving short fiber suspensions: numerical difficulties and efficient micro-macro solvers. Arch. Comput. Meth. Eng. 2009;16(1):1-30.

[16] Prulière E, Férec J, Chinesta F, Ammar A. An efficient reduced simulation of residual stresses in composite forming processes. Int. J. Mater. Form. 2010;3(2):1339-50.

[17] Reddy JN, Robbins Jr DH. Theories and computational models for composite laminates. Appl. Mech. Rev. 1994;47(6):147-67.

[18] Ren JG. Exact solutions for laminated cylindrical shells in cylindrical bending. Compos. Sci. Technol. 1987;29:169-87.

[19] Ryckelynck D. Hyper reduction of mechanical models involving internal variables. Int. J. Numer. Methods Eng. 2009;77(1):75-89.

[20] Ryckelynck D, Chinesta F, Cueto E, Ammar A. On the a priori model reduction: overview and recent developments. Arch. Comput. Meth. Eng. 2006;13(1):91-128.

[21] Vidal P, Gallimard L, Polit O. Assessment of a composite beam finite element based on the proper generalized decomposition. Compos. Struct. 2012;94(5):1900-10. 